Research Article

\title{
Clinical Utility of Serum Holotranscobalamin Measurements in Patients with First-Ever Ischemic Stroke
}

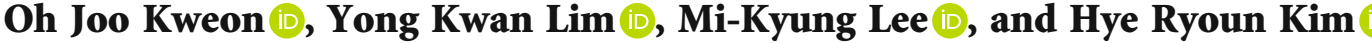 \\ Department of Laboratory Medicine, Chung-Ang University College of Medicine, Seoul, Republic of Korea \\ Correspondence should be addressed to Hye Ryoun Kim; hyekim@cau.ac.kr
}

Received 19 March 2021; Revised 24 August 2021; Accepted 28 August 2021; Published 11 September 2021

Academic Editor: Dr Shabana

Copyright (C) 2021 Oh Joo Kweon et al. This is an open access article distributed under the Creative Commons Attribution License, which permits unrestricted use, distribution, and reproduction in any medium, provided the original work is properly cited.

\begin{abstract}
Background. Whether holotranscobalamin (holoTC) indicates B12 deficiency more sensitively than total vitamin B12 (B12) is unclear. This study is aimed at determining the impact of serum holoTC level as a risk factor for ischemic stroke and investigating its association with disease severity and short-term outcomes. Methods. Serum holoTC, total B12, and homocysteine levels were compared between 130 stroke patients and 138 healthy controls. Biomarker level correlations with disease severity and stroke functional outcomes were investigated. Results. holoTC levels were lower and homocysteine levels were higher in stroke patients than in healthy controls $(P<0.05)$. The holoTC/total B12 ratio and homocysteine level significantly predicted ischemic stroke in the multivariable regression analysis $(P<0.05)$. Along with hyperhomocysteinemia, patients more often had holoTC than total B12 deficiency (6.2\% vs. 3.1\%). holoTC levels negatively correlated with homocysteine levels (partial $R$ $-0.165, P<0.05)$ in stroke patients in multiple linear regression analyses, but not total B12 levels. The holoTC level and holoTC/total B12 ratio, but not homocysteine and total B12 levels, negatively correlated with the National Institute of Health Stroke Scale (partial $R,-0.405$ and -0.207 , respectively, $P<0.01$ ). Conclusions. Measurements of serum holoTC levels combined with total B12 and homocysteine levels may provide valuable information for predicting ischemic stroke and its severity and short-term outcomes of ischemic stroke patients.
\end{abstract}

\section{Introduction}

Stroke is the second most common cause of death worldwide and is the leading cause of acquired disability in adults in most regions [1]. Ischemic stroke has potentially treatable contributory factors such as hypertension, diabetes mellitus, cardiac diseases, smoking, alcohol intake, obesity, and hyperlipidemia $[2,3]$. In addition to these, inadequate vitamin B12 or cobalamin status has emerged as a new treatable factor contributing to stroke risk, implying that early detection of vitamin B12 deficiency is crucial $[4,5]$.

Vitamin B12 is essential for cellular metabolism, particularly for DNA synthesis and both fatty acid and amino acid metabolism. Importantly, vitamin B12 is a cofactor in homocysteine metabolism, and its deficiency may be an important cause of hyperhomocysteinemia $[6,7]$, which is closely correlated to the risk of ischemic stroke as a result of the increased development of carotid plaques [2]. Vitamin B12 supplementation may contribute to stroke prevention [8], thus lowering homocysteine levels.

Currently, vitamin B12 status is mainly determined by measuring total levels of vitamin B12 in the serum (total B12), but the diagnostic accuracy of these tests is not satisfactory for detecting vitamin B12 deficiency $[4,9]$. This could be because total B12 assays detect both active and inert forms of vitamin B12. Only $20-25 \%$ of serum total B12 is in the active form, i.e., holotranscobalamin (holoTC). holoTC is vitamin B12 that is bound to the serum transport protein transcobalamin for delivery to cells for metabolism. Total B12 measurement can, therefore, be insensitive in detecting functional vitamin B12 deficiency $[10,11]$. Another potentially accurate way to diagnose the functional adequacy of vitamin B12 is to measure the level of holoTC $[9,12]$. However, it remains controversial whether holoTC values 
provide an earlier or more sensitive indication of vitamin B12 deficiency than total B12 values as the results of different studies have been inconsistent [11].

The clinical usefulness of total B12 measurements in ischemic stroke patients has been assessed previously [2]; however, few studies have investigated the clinical utility of holoTC measurements for this patient population. This study is aimed at determining the impact of serum holoTC level as a risk factor for ischemic stroke and investigating its association with disease severity and short-term outcomes, focusing on the advantages, if any, of holoTC measurements over total B12 measurements.

\section{Materials and Methods}

2.1. Ethics Statements. This prospective case-control study was approved by the Chung-Ang University Hospital Institutional Review Board (IRB), and the need for informed consent was waived according to the IRB policy (IRB approval number 1712-022-16129).

2.2. Study Subjects. The study included first-ever ischemic stroke patients who experienced acute neurologic symptoms with symptom onset within $24 \mathrm{~h}$ and visited the emergency room of Chung-Ang University Hospital, Seoul, Republic of Korea, from August 2018 to February 2019. A total of 130 stroke patients (81 males) were included (Figure 1). The diagnosis of ischemic stroke was confirmed based on findings from diffuse brain magnetic resonance imaging (MRI) according to the American Heart Association/American Stroke Association definition [13]. In addition to the stroke patients, 138 (88 males) healthy individuals who visited the hospital for health check-ups were enrolled in the study as healthy controls. The healthy controls were screened for the absence of stroke using brain MRI.

Patients diagnosed with hemorrhagic stroke, who previously had an injury to the central nervous system or who experienced cerebro- or cardiovascular ischemic events, were excluded from the study. To limit factors affecting the circulating homocysteine level, patients with chronic kidney disease and receiving certain drugs such as fibrates, metformin, and methotrexate were excluded. Patients who took therapeutic vitamin B12 supplements for other diseases such as gastric cancer were also excluded. Patients who had been taking nutritional dietary supplements such as vitamin B12 before the onset of stroke were not excluded and their B12 supplementation was discontinued during their hospitalization period.

2.3. Laboratory Data. Serum levels of holoTC, total B12, homocysteine, hemoglobin A1c (HbAlc), fasting glucose, and lipids (total cholesterol, LDL cholesterol, and triglycerides) were measured using the blood samples obtained from ischemic patients at the time of diagnosis and obtained from healthy controls during their visit for health check-ups. holoTC levels were assessed using an Abbott Architect Active-B12 (holotranscobalamin) assay (Abbott Laboratories, Abbott Park, IL, USA) with an ARCHITECT i2000SR (Abbott Laboratories), which is an automated chemilumi- nescent microparticle immunoassay analyzer. Total B12 levels were measured using radioimmunoassay (MP Biomedicals, Orangeburg, NY, USA). The holoTC/total B12 ratio was calculated after unifying the units and was expressed as a percentage. Homocysteine, fasting glucose, and lipid profile levels were assessed using the Beckman Coulter AU5800 clinical chemistry analyzer (Beckman Coulter, Brea, CA, USA). HbAlc levels were measured using the VARIANT II TURBO Hemoglobin Testing System (Bio-Rad Laboratories, Hercules, CA, USA). All analyses were performed according to the instructions from the respective assay manufacturers.

2.4. Clinical Data. To assess disease severity and functional outcomes of stroke patients, medical records from when the patient was admitted were collected and reviewed. The following data were collected: symptoms; body mass index (BMI); admission National Institute of Health Stroke Scale (NIHSS) scores, which reflect disease severity; and modified Rankin Scale (mRS) scores at the time of discharge, which reflect the functional outcome.

2.5. Statistical Analysis. The Kolmogorov-Smirnov test was conducted to assess the normality of data distribution. Laboratory or clinical data were compared between the study groups using one-way analysis of variance or independent Student's $t$-tests for variables normally distributed and using the Kruskal-Wallis test or Mann-Whitney $U$ test for variables not normally distributed. Mann-Whitney $U$ tests or Scheffe's multiple comparison tests were conducted for post hoc analysis. Univariable and multivariable logistic regression analyses were conducted to identify biomarkers that could predict ischemic stroke events, while multiple linear regression analysis was used to investigate relationships between laboratory and clinical data. In the logistic regression and multiple linear regression analyses, possible confounding factors such as age; BMI; and serum levels of $\mathrm{HbA1c}$, fasting glucose, and lipids were included, along with serum levels of holoTC, total B12, and homocysteine and clinical scores, to adjust for confounding effects. Statistical analysis was performed using SPSS software, version 19 (SPSS, Chicago, IL), and $P<0.05$ was considered statistically significant.

\section{Results}

3.1. Clinical Characteristics of Stroke Patients. The clinical characteristics of the 130 first-ever ischemic stroke patients are listed in Table 1. The median age of stroke patients was 71 years (interquartile range (IQR), 59-78 years; age range, 44-89 years). The median ages for male and female patients were 67 years (IQR: 58-75 years) and 69 years (IQR, 61-80 years), respectively. The most frequent symptom was leftside hemiparesis/hemiplegia (31.5\%), and the most frequently involved brain region was the frontal lobe $(23.1 \%)$. The median NIHSS scores of the total, male, and female stroke patients were 2 (IQR, 1-5.25; $\min 0, \max 31), 3$ (IQR, 1-5.5; $\min 0$, $\max 31$ ), and 2 (IQR, 0.5-5.5; min 0, $\max 27)$, respectively. The median $\mathrm{mRS}$ scores of the total, 


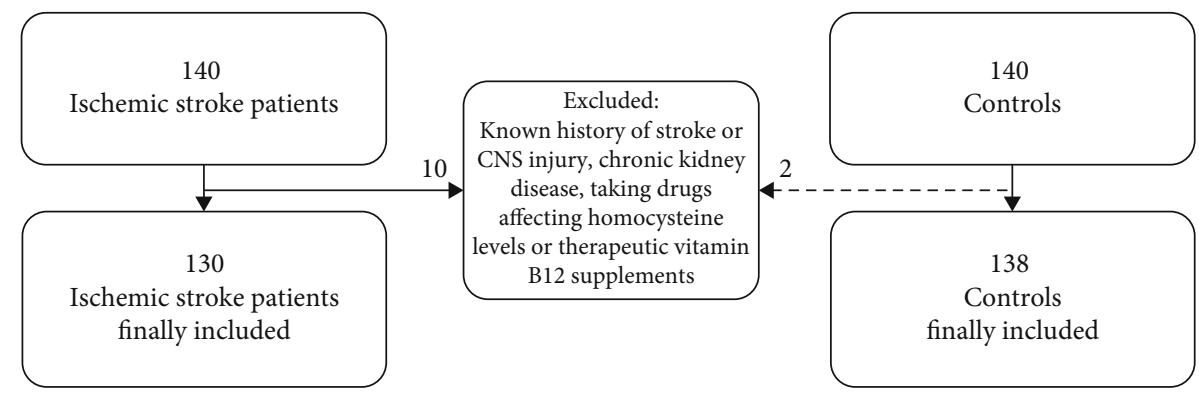

FIGURE 1: Flowchart of the inclusion and exclusion of the study subjects. In total, 130 ischemic stroke patients and 138 normal controls were included in this study. Patients who previously had an injury to the central nervous system or who had experienced stroke events were excluded. Patients taking therapeutic vitamin B12 supplements for other diseases such as gastric cancer and with factors affecting serum homocysteine levels (chronic kidney disease and certain drugs) were also excluded.

TABLE 1: Characteristics of the first-ever stroke patients in this study.

\begin{tabular}{lc}
\hline Characteristics & $\begin{array}{c}\text { Patients } \\
\text { with ischemic stroke } \\
(N=130)\end{array}$ \\
\hline Age, median years [Q1-Q3] & $71[59-78]$ \\
Sex, \% male $(N)$ & $62.3(81)$ \\
Clinical presentations, \% $N)$ & \\
Hemiparesis/hemiplegia/weakness & \\
Left & $31.5(41)$ \\
Right & $16.2(21)$ \\
Bilateral & $1.5(2)$ \\
Dysarthria & $27.7(36)$ \\
Dizziness & $16.2(21)$ \\
Mental change & $10.0(13)$ \\
Amnesia & $1.5(2)$ \\
Involved brain region, \% $(N)$ & \\
Frontal lobe & $23.1(30)$ \\
Temporal lobe & $22.3(29)$ \\
Parietal lobe & $16.2(21)$ \\
Occipital lobe & $12.3(16)$ \\
Basal ganglia & $14.6(19)$ \\
Corona radiata & $13.8(18)$ \\
Pons & $13.1(17)$ \\
Cerebellum & $17.7(23)$ \\
Brain atrophy, \% $N)$ & $6.9(9)$ \\
NIHSS, median [Q1-Q3] & $2[1-5.25]$ \\
mRS, median [Q1-Q3] & $4[2-4]$ \\
\hline Abbrevtion & \\
&
\end{tabular}

Abbreviations: NIHSS: National Institute of Health Stroke Scale; mRS: modified Rankin Scale.

male, and female stroke patients were 4 (IQR, 2-4; min 1, max 5), 4 (IQR, 3-4; $\min 1$, $\max 5$ ), and 4 (IQR, 2-4; min $1, \max 5)$, respectively. There were no statistically significant differences in the NIHSS and mRS scores between the sexes.

There were no significant differences in age between the sexes in either group or between healthy controls and stroke patients $(P \geq 0.05)$. The median age of healthy controls was 69.8 years (IQR, 62-76 years; range, $46-96$ years). The median ages of male and female healthy controls were 67.5 years (IQR, 60-74.5 years) and 70 years (IQR, 64-77 years), respectively.

3.2. holoTC, Total B12, and Homocysteine Levels of Study Subjects. Laboratory profiles, including measurements of holoTC, total B12, and homocysteine levels, of stroke patients and healthy controls are listed in Table 2. Absolute serum holoTC levels and holoTC/total B12 ratios were significantly lower in stroke patients than in healthy controls (median: 127.2 vs. $167.4 \mathrm{pmol} / \mathrm{L}$ and $33.7 \%$ vs. $38.3 \%$, respectively, $P<0.01$ for both). However, no difference was found in total B12 levels between the two groups $(P>0.05$ ). Homocysteine levels were significantly higher in stroke patients than in healthy controls $(12.3$ vs. $10.2 \mu \mathrm{mol} / \mathrm{L}, P<$ $0.01)$.

The proportions of patients with holoTC and total B12 levels below the cut-off values $(<37 \mathrm{pmol} / \mathrm{L}$ and $<200 \mathrm{pg} / \mathrm{mL}$, respectively) and with hyperhomocysteinemia ( $>14 \mu \mathrm{mol} / \mathrm{L})$ were higher in the stroke group than in the control group (holoTC, $6.9 \%$ vs. $1.4 \%$; total B12, 10.0 vs. $0.7 \%$; homocysteine, $28.4 \%$ vs. $5.1 \%$, respectively; all $P<0.05)$. HbAlc and fasting glucose levels and BMI also differed significantly between the groups $(P<0.05)$.

3.3. Logistic Regression Analysis for Predicting Ischemic Stroke. The results of the logistic regression analysis are listed in Table 3. In the univariable logistic regression analysis, holoTC, total B12, holoTC/total B12 ratio, homocysteine, $\mathrm{HbAlc}$, fasting glucose, and BMI showed significant $\beta$ values $(P<0.05)$. Among them, low holoTC/total B12 ratio, high homocysteine and fasting glucose levels, and BMI were statistically significant predictors of ischemic stroke $(P<0.05)$ in the multivariable logistic regression analysis.

3.4. Correlations of holoTC and Total B12 Levels with Serum Homocysteine Levels. In Spearman's rank tests, only holoTC levels showed a statistically significant negative correlation with homocysteine levels $\left(r_{s},-0.196, P=0.025\right)$ in stroke patients. However, in healthy controls, both holoTC and total B12 levels had significant inverse correlations with serum homocysteine levels $\left(r_{s},-0.212\right.$ and $-0.321 ; P=0.012$ and $P<0.01$, respectively). The holoTC/total B12 ratio did not show a significant correlation with homocysteine levels 


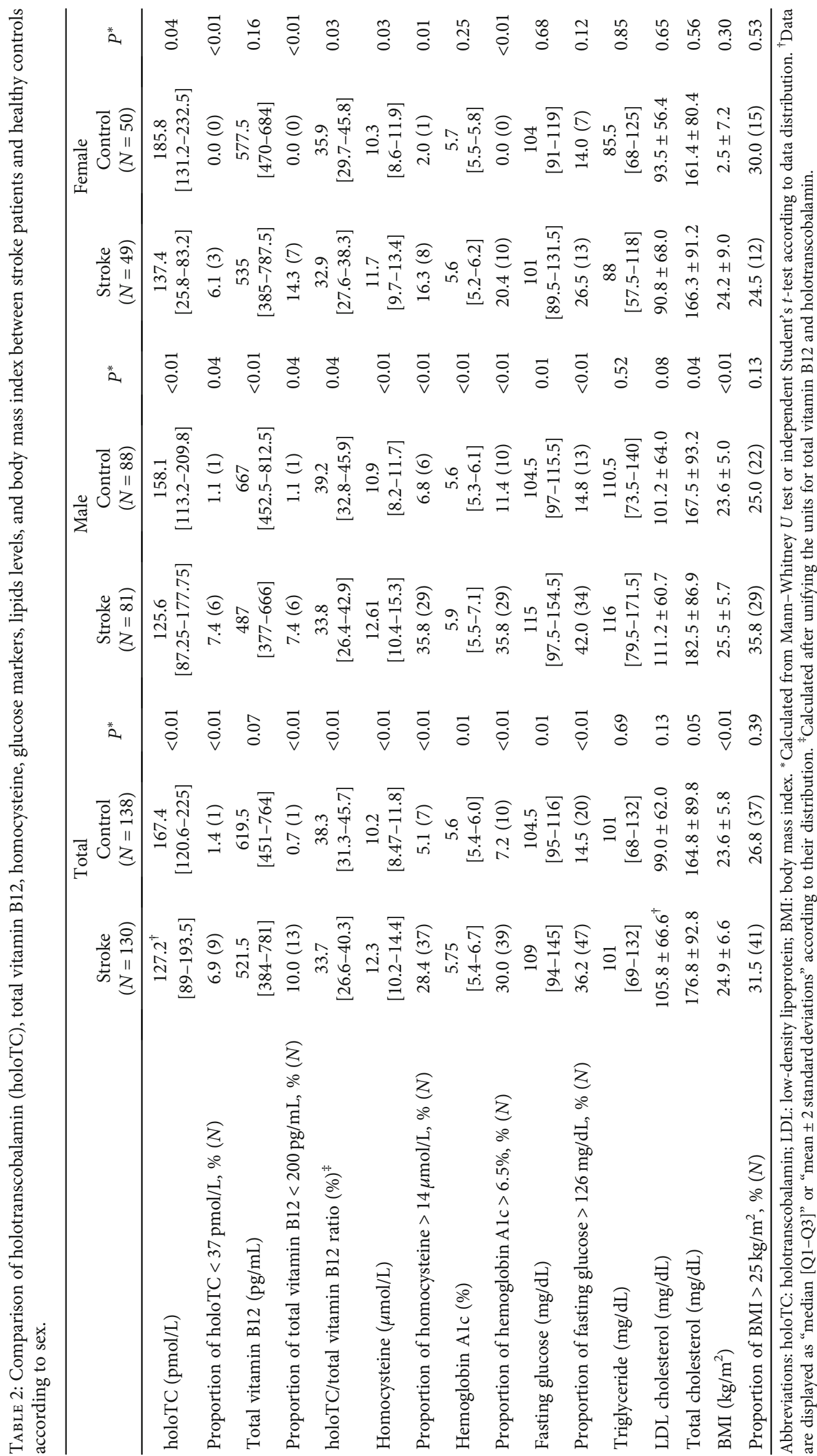


TABLE 3: Logistic regression analysis results for predicting ischemic stroke.

\begin{tabular}{|c|c|c|c|c|c|c|}
\hline \multirow[t]{2}{*}{ Variables } & \multicolumn{3}{|c|}{ Univariable } & \multicolumn{3}{|c|}{ Multivariable } \\
\hline & $\beta$ coefficient (SE) & OR $(95 \% \mathrm{CI})$ & $P$ & $\beta$ coefficient (SE) & OR $(95 \% \mathrm{CI})$ & $P$ \\
\hline holoTC (pmol/L) & $-0.004(0.002)$ & $0.996(0.993-0.999)$ & $<0.01$ & - & - & $>0.05$ \\
\hline Total vitamin $\mathrm{B} 12$ (pg/mL) & $-0.001(0.001)$ & $0.999(0.998-1.000)$ & 0.01 & - & - & $>0.05$ \\
\hline holoTC/total vitamin B12 (\%)* & $-0.410(0.011)$ & $0.960(0.938-0.981)$ & $<0.01$ & $-0.450(0.013)$ & $0.956(0.932-0.981)$ & $<0.01$ \\
\hline Homocysteine $(\mu \mathrm{mol} / \mathrm{L})$ & $0.270(0.049)$ & $1.310(1.190-1.442)$ & $<0.01$ & $0.251(0.051)$ & $1.285(1.163-1.421)$ & $<0.01$ \\
\hline Hemoglobin Alc (\%) & $0.503(0.133)$ & $1.653(1.274-2.144)$ & $<0.01$ & - & - & $>0.05$ \\
\hline Fasting glucose (mg/dL) & $0.014(0.004)$ & $1.014(1.006-1.021)$ & $<0.01$ & $0.015(0.004)$ & $1.015(1.007-1.024)$ & $<0.01$ \\
\hline Triglyceride (mg/dL) & - & - & $>0.05$ & - & - & - \\
\hline LDL cholesterol (mg/dL) & - & - & $>0.05$ & - & - & - \\
\hline Total cholesterol (mg/dL) & - & - & $>0.05$ & - & - & - \\
\hline BMI $\left(\mathrm{kg} / \mathrm{m}^{2}\right)$ & $0.152(0.042)$ & $1.164(1.073-1.263)$ & $<0.01$ & $0.118(0.046)$ & $1.125(1.029-1.231)$ & 0.01 \\
\hline
\end{tabular}

Abbreviations: SE: standard error; CI: confidence interval; holoTC: holotranscobalamin; LDL: low-density lipoprotein; BMI: body mass index. *Calculated after unifying the units for total vitamin B12 and holotranscobalamin.

TABLE 4: Association of metabolic variables with serum homocysteine levels and NIHSS by multiple linear regression analyses in 130 patients with first-ever ischemic stroke.

\begin{tabular}{|c|c|c|c|c|c|c|}
\hline \multirow[t]{2}{*}{ Variables } & \multicolumn{3}{|c|}{ Homocysteine $(\mu \mathrm{mol} / \mathrm{L})$} & \multicolumn{3}{|c|}{ NIHSS } \\
\hline & $\beta(\mathrm{SE})$ & Partial $R$ & $P$ & $\beta(\mathrm{SE})$ & Partial $R$ & $P$ \\
\hline holoTC (pmol/L)* & $-0.107(0.054)$ & -0.165 & 0.048 & $-0.830(0.156)$ & -0.405 & $<0.01$ \\
\hline Total vitamin $\mathrm{B} 12(\mathrm{pg} / \mathrm{mL})^{*}$ & - & - & - & - & - & - \\
\hline holoTC/total vitamin B12 (\%), & - & - & - & $-0.631(0.233)$ & -0.207 & $<0.01$ \\
\hline Homocysteine $(\mu \mathrm{mol} / \mathrm{L})^{*}$ & & & & - & - & - \\
\hline Hemoglobin Alc $(\%)^{*}$ & - & - & - & - & - & - \\
\hline Fasting glucose $(\mathrm{mg} / \mathrm{dL})^{*}$ & - & - & - & $0.532(0.223)$ & 0.182 & 0.019 \\
\hline Triglyceride $(\mathrm{mg} / \mathrm{dL})^{*}$ & - & - & - & - & - & - \\
\hline LDL cholesterol (mg/dL) & - & - & - & - & - & - \\
\hline Total cholesterol (mg/dL) & $0.004(0.001)$ & 0.179 & 0.040 & - & - & - \\
\hline BMI $\left(\mathrm{kg} / \mathrm{m}^{2}\right)$ & - & - & - & $0.022(0.011)$ & 0.158 & 0.041 \\
\hline Age (years) ${ }^{*}$ & - & - & - & $0.006(0.003)$ & 0.146 & 0.049 \\
\hline
\end{tabular}

Abbreviations: SE: standard error; holoTC: holotranscobalamin; LDL: low-density lipoprotein; BMI: body mass index. ${ }^{*}$ Log-transformed values were used for analysis. ${ }^{\dagger}$ Calculated after unifying the units for total vitamin B12 and holotranscobalamin.

in patients or healthy controls (both $P>0.05$ ). Among stroke patients, more patients had holoTC deficiency with hyperhomocysteinemia $(6.2 \%, 8 / 130)$ than total B12 deficiency with hyperhomocysteinemia $(3.1 \%, 4 / 130)$.

The multiple linear regression analysis also revealed that holoTC levels had a statistically significant negative correlation with homocysteine levels in patients with stroke ( $\beta-0.107, P<0.05)$, but total $\mathrm{B} 12$ and holoTC/total B12 levels did not (Table 4$)$. The serum total cholesterol level was positively correlated to homocysteine levels $(P<0.05)$.

3.5. Correlations of holoTC, Total B12, and Homocysteine Levels with Functional Scale Scores. Both holoTC and total B12 levels showed significant $r_{\mathrm{s}}$ values for correlation with NIHSS and mRS scores in Spearman's rank correlation tests after adjusting for HbAlc, fasting glucose, and total cholesterol levels. The $r_{s}$ values of the correlation of holoTC and total B12 levels with the NIHSS score were
-0.29 and -0.28 (both $P<0.01$ ), respectively, and with the mRS scores were -0.31 and -0.29 (both $P<0.01$ ), respectively. Homocysteine levels and holoTC/total B12 ratios did not correlate significantly with either of the functional scales $(P>0.05)$.

In contrast, multiple linear regression analyses showed that the holoTC level and holoTC/total B12 ratio were independently and negatively correlated to NIHSS scores $(\beta$, -0.830 and -0.631 ; partial $R,-0.405$ and -0.207 , respectively, $P<0.01$ for both), but total B12 and homocysteine levels were not. Fasting glucose, BMI, and patient's age were also associated with NIHSS scores (Table 4). In a subgroup analysis based on the NIHSS score, both holoTC and total B12 levels showed significantly decreased values in the "NIHSS 21-42" subgroup ("severe stroke" patients) compared to any other group (Table 5). The holoTC/total B12 ratio and homocysteine levels were not statistically significantly different among the subgroups. In the mRS subgroup analysis, 
TABLE 5: Holotranscobalamin (holoTC), total vitamin B12, homocysteine, glucose markers, lipids levels, and body mass index according to the National Institute of Health Stroke Scale (NIHSS).

\begin{tabular}{|c|c|c|c|c|c|c|c|}
\hline & $\begin{array}{c}0 \\
(N=26)\end{array}$ & $\begin{array}{c}1-4 \\
(N=65)\end{array}$ & $\begin{array}{c}\text { NIHSS } \\
5-15 \\
(N=29)\end{array}$ & $\begin{array}{c}16-20 \\
(N=4)\end{array}$ & $\begin{array}{c}21-42 \\
(N=6)\end{array}$ & $P^{*}$ & $\begin{array}{c}\text { Kruskal-Wallis } \\
\text { grouping }^{\dagger}\end{array}$ \\
\hline holoTC (pmol/L) & $\begin{array}{c}143.7^{\ddagger} \\
{[73.0-184.8]}\end{array}$ & $\begin{array}{c}127.0 \\
{[102.8-196.5]}\end{array}$ & $\begin{array}{c}118 \\
{[77.2-178.8]}\end{array}$ & $\begin{array}{c}141 \\
{[117-156]}\end{array}$ & $\begin{array}{c}58.1 \\
{[32.0-62.4]}\end{array}$ & 0.04 & $\mathrm{a} / \mathrm{b} / \mathrm{b} / \mathrm{a} / \mathrm{c}$ \\
\hline Total vitamin B12 (pg/mL) & $\begin{array}{c}496.5 \\
{[280.0-679.5]}\end{array}$ & $\begin{array}{c}558 \\
{[440-819.3]}\end{array}$ & $\begin{array}{c}480 \\
{[299.0-756.0]}\end{array}$ & $\begin{array}{c}393 \\
{[277-566]}\end{array}$ & $\begin{array}{c}194 \\
{[162-290]}\end{array}$ & $<0.01$ & $\mathrm{a} / \mathrm{b} / \mathrm{a} / \mathrm{c} / \mathrm{d}$ \\
\hline holoTC/total vitamin B12 $(\%)^{\S}$ & $\begin{array}{c}34.0 \\
{[30.8-41.9]}\end{array}$ & $\begin{array}{c}30.6 \\
{[24.7-38.4]}\end{array}$ & $\begin{array}{c}34.5 \\
{[30.4-42.4]}\end{array}$ & $\begin{array}{c}43.7 \\
{[26.1-62.2]}\end{array}$ & $\begin{array}{c}29.5 \\
{[27.9-48.0]}\end{array}$ & 0.143 & \\
\hline Homocysteine $(\mu \mathrm{mol} / \mathrm{L})$ & $\begin{array}{c}11.7 \\
{[9.7-13.3]}\end{array}$ & $\begin{array}{c}12.6 \\
{[10.8-14.7]}\end{array}$ & $\begin{array}{c}12.8 \\
{[9.7-14.4]}\end{array}$ & $\begin{array}{c}13 \\
{[11.0-15.8]}\end{array}$ & $\begin{array}{c}10.9 \\
{[10.4-11.2]}\end{array}$ & 0.26 & - \\
\hline Hemoglobin A1c (\%) & $\begin{array}{c}5.5 \\
{[2.3-5.7]}\end{array}$ & $\begin{array}{c}5.9 \\
{[5.5-6.9]}\end{array}$ & $\begin{array}{c}6.3 \\
{[5.6-7.4]}\end{array}$ & $\begin{array}{c}6 \\
{[5.5-6.0]}\end{array}$ & $\begin{array}{c}5.5 \\
{[5.0-6.2]}\end{array}$ & 0.02 & $\mathrm{a} / \mathrm{b} / \mathrm{b} / \mathrm{b} / \mathrm{a}$ \\
\hline Fasting glucose (mg/dL) & $\begin{array}{c}100.0 \\
{[92.3-110.0]}\end{array}$ & $\begin{array}{c}109.0 \\
{[94-135.8]}\end{array}$ & $\begin{array}{c}112.0 \\
{[99.0-159.0]}\end{array}$ & $\begin{array}{c}130 \\
{[112.0-147.8]}\end{array}$ & $\begin{array}{c}189.5 \\
{[156.5-209.0]}\end{array}$ & 0.04 & $\mathrm{a} / \mathrm{a} / \mathrm{a} / \mathrm{a} / \mathrm{b}$ \\
\hline Triglyceride (mg/dL) & $\begin{array}{c}81 \\
{[54.0-107.5]}\end{array}$ & $\begin{array}{c}116 \\
{[79.0-171.0]}\end{array}$ & $\begin{array}{c}101.0 \\
{[52.0-129.0]}\end{array}$ & $\begin{array}{c}102 \\
{[53.5-160.5]}\end{array}$ & $\begin{array}{c}108.5 \\
{[69.8-145.8]}\end{array}$ & 0.11 & - \\
\hline LDL cholesterol (mg/dL) & $101.7 \pm 31.6^{\ddagger}$ & $103.5 \pm 36.02$ & $106.5 \pm 31.7$ & $85.8 \pm 34.5$ & $108.8 \pm 21.5$ & 0.71 & - \\
\hline Total cholesterol (mg/dL) & $174.0 \pm 85.9$ & $181.6 \pm 83.6$ & $174.6 \pm 88.9$ & $164.3 \pm 69.6$ & $169.2 \pm 58.9$ & 0.64 & - \\
\hline BMI $\left(\mathrm{kg} / \mathrm{m}^{2}\right)$ & $25.1 \pm 6.7$ & $25.3 \pm 6.9$ & $24.3 \pm 5.0$ & $22.3 \pm 7.2$ & $27.0 \pm 8.7$ & 0.57 & - \\
\hline
\end{tabular}

Abbreviations: LDL: low-density lipoprotein; BMI: body mass index. *Calculated from the one-way analysis of variance or the Kruskal-Wallis test, according to data distribution. ${ }^{\dagger}$ The same letters indicate the nonsignificant difference between groups based on the Mann-Whitney $U$ test. ${ }^{\ddagger}$ Data are displayed as "median [Q1-Q3]" or “mean \pm 2 standard deviations" according to their distribution. ${ }^{\S \ddagger}$ Calculated after unifying the units for total vitamin B12 and holotranscobalamin.

holoTC levels and total B12 levels were significantly decreased in the "mRS score 5" subgroup compared to any other group (Table 6).

\section{Discussion}

The study findings revealed that the holoTC/total B12 ratio could be a predictive factor for ischemic stroke. holoTC measurements reflected homocysteine levels better than total B12 measurements in stroke patients; thus, holoTC measurements in ischemic stroke patients may be useful for identifying patients who have vitamin B12 deficiencyrelated hyperhomocysteinemia. In addition, holoTC levels showed significant associations with stroke severity and functional outcomes. To our best knowledge, this was the first case-control study that investigated the diagnostic or prognostic utility of serum holoTC measurements in patients with ischemic stroke.

Vitamin B12 is a cofactor in homocysteine metabolism, and homocysteine can mediate the development of cerebrovascular disease. This can occur through several different mechanisms, including its adverse effects on vascular endothelium and smooth muscle cells, which cause alterations in subclinical arterial structure and function [14-16]. Systematic reviews of observational studies have shown a strong and positive association between serum homocysteine levels and stroke events $[5,17,18]$, consistent with our results. Irrespective of increased homocysteine levels, vitamin B12 deficiency has been shown to impair bone metabolism through reduced levels of taurine, an amino acid that is considered to reduce the risks of atherosclerosis and cardiovascular disease, and has shown neuroprotective effects in animal stroke models $[9,19,20]$.

Transcobalamin is essential for the transport of vitamin B12 from the intestine into most cells of the body, and patients lacking this protein eventually develop vitamin B12 deficiency [6]. When vitamin B12 is bound to transcobalamin, it is termed holoTC and represents the biologically active fraction of vitamin B12 that can be delivered to all DNA-synthesizing cells. In the absence of a gold standard to detect vitamin B12 deficiency $[4,11]$, current laboratory tests, which measure total B12, may fail to diagnose the deficiency in the early stage $[6,21,22]$. In 1987, Victor Herbert proposed a model for the staged development of vitamin B12 deficiency, of which holoTC is the first indicator. Based on this model, several studies have confirmed that serum total B12 is a relatively poor marker, with low sensitivity and specificity, for predicting vitamin B12 status, and that instead holoTC may be a useful and sensitive diagnostic indicator of vitamin B12 status [4, 6, 23]. However, Herbert's model for the development of vitamin B12 deficiency is controversial, and an alternative model reflecting the enterohepatic recycling regulation of holoTC was proposed by Golding [11] who argued that holoTC cannot be used to assess vitamin B12 status any more reliably than total B12.

Few studies have investigated holoTC measurements in ischemic stroke patients. One previous study investigated the efficacy of holoTC measurements in ischemic cerebrovascular 
TABLE 6: Holotranscobalamin (holoTC), total vitamin B12, homocysteine, glucose markers, lipids levels, and body mass index levels according to the modified Rankin Scale (mRS).

\begin{tabular}{|c|c|c|c|c|c|c|c|}
\hline & $\begin{array}{c}1 \\
(N=13)\end{array}$ & $\begin{array}{c}2 \\
(N=21)\end{array}$ & $\begin{array}{c}\mathrm{mRS} \\
3 \\
(N=17)\end{array}$ & $\begin{array}{c}4 \\
(N=56)\end{array}$ & $\begin{array}{c}5 \\
(N=23)\end{array}$ & $P^{*}$ & $\begin{array}{c}\text { Kruskal-Wallis } \\
\text { grouping }^{\dagger}\end{array}$ \\
\hline holoTC (pmol/L) & $\begin{array}{c}139.6^{\ddagger} \\
{[102.8-200.0]}\end{array}$ & $\begin{array}{c}137.4 \\
{[108.2-207.2]}\end{array}$ & $\begin{array}{c}108.0 \\
{[84.4-} \\
190.1]\end{array}$ & $\begin{array}{c}109.1 \\
{[75.7-176.2]}\end{array}$ & $\begin{array}{c}72.7 \\
{[58.1-128.1]}\end{array}$ & $<0.01$ & $\mathrm{a} / \mathrm{a} / \mathrm{a} / \mathrm{a} / \mathrm{b}$ \\
\hline Total vitamin B12 (pg/mL) & $\begin{array}{c}558.0 \\
{[376.0-814.0]}\end{array}$ & $\begin{array}{c}556.0 \\
{[475.3-787.8]}\end{array}$ & $\begin{array}{c}430.0 \\
{[361.0-} \\
770.5]\end{array}$ & $\begin{array}{c}486.0 \\
{[288.0-} \\
631.0]\end{array}$ & $\begin{array}{c}302.0 \\
{[86.5-502.5]}\end{array}$ & $<0.01$ & $\mathrm{a} / \mathrm{a} / \mathrm{a} / \mathrm{a} / \mathrm{b}$ \\
\hline holoTC/total vitamin B12 (\%) & $\begin{array}{c}35.6 \\
{[29.5-42.6]}\end{array}$ & $\begin{array}{c}31.8 \\
{[27.8-38.3]}\end{array}$ & $\begin{array}{c}33.6 \\
{[28.7-37.6]}\end{array}$ & $\begin{array}{c}33.9 \\
{[25.2-43.3]}\end{array}$ & $\begin{array}{c}31.0 \\
{[26.0-40.8]}\end{array}$ & 0.84 & - \\
\hline Homocysteine $(\mu \mathrm{mol} / \mathrm{L})$ & $\begin{array}{c}11.2 \\
{[10.0-17.4]}\end{array}$ & $\begin{array}{c}12.8 \\
{[9.8-13.9]}\end{array}$ & $\begin{array}{c}13.3 \\
{[10.9-15.1]}\end{array}$ & $\begin{array}{c}12.3 \\
{[10.2-14.7]}\end{array}$ & $\begin{array}{c}11.9 \\
{[9.8-14.6]}\end{array}$ & 0.94 & - \\
\hline Hemoglobin A1c (\%) & $\begin{array}{c}5.7 \\
{[5.1-7.6]}\end{array}$ & $\begin{array}{c}6.1 \\
{[5.4-7.0]}\end{array}$ & $\begin{array}{c}6.1 \\
{[5.6-8.0]}\end{array}$ & $\begin{array}{c}5.8 \\
{[5.3-6.7]}\end{array}$ & $\begin{array}{c}5.7 \\
{[5.4-6.4]}\end{array}$ & 0.44 & - \\
\hline Fasting glucose (mg/dL) & $\begin{array}{c}143.0 \\
{[104.0-207.0]}\end{array}$ & $\begin{array}{c}107.0 \\
{[92.0-150.5]}\end{array}$ & $\begin{array}{c}123.0 \\
{[104.0-} \\
161.0]\end{array}$ & $\begin{array}{c}107.0 \\
{[91.0-137.8]}\end{array}$ & $\begin{array}{c}105.0 \\
{[96.0-130.0]}\end{array}$ & 0.14 & - \\
\hline Triglyceride (mg/dL) & $\begin{array}{c}60.0 \\
{[52.0-142.0]}\end{array}$ & $\begin{array}{c}88.0 \\
{[50.5-135.5]}\end{array}$ & $\begin{array}{l}104.0 \\
{[76.0-} \\
143.0]\end{array}$ & $\begin{array}{c}99.5 \\
{[70.0-183.8]}\end{array}$ & $\begin{array}{c}112.0 \\
{[88.0-171.0]}\end{array}$ & 0.40 & - \\
\hline LDL cholesterol (mg/dL) & $107.4 \pm 76.4^{\ddagger}$ & $94.3 \pm 67.4$ & $108.5 \pm 46.3$ & $105.8 \pm 74.8$ & $100.1 \pm 52.1$ & 0.67 & - \\
\hline Total cholesterol (mg/dL) & $178.5 \pm 95.4$ & $159.1 \pm 102.1$ & $181.5 \pm 67.9$ & $181.2 \pm 101.7$ & $167.6 \pm 72.6$ & 0.42 & - \\
\hline BMI $\left(\mathrm{kg} / \mathrm{m}^{2}\right)$ & $24.7 \pm 3.2$ & $24.3 \pm 8.2$ & $23.9 \pm 4.6$ & $25.7 \pm 6.9$ & $25.0 \pm 5.5$ & 0.17 & - \\
\hline
\end{tabular}

Abbreviations: LDL: low-density lipoprotein; BMI: body mass index. ${ }^{*}$ Calculated from the one-way analysis of variance or the Kruskal-Wallis test according to data distribution. ${ }^{\dagger}$ The same letters indicate the nonsignificant difference between groups based on the Mann-Whitney $U$ test. ${ }^{*}$ Data are displayed as "median [Q1, Q3]" or "mean \pm 2 standard deviations" according to their distribution. ${ }^{\S \neq}$ Calculated after unifying the units for total vitamin B12 and holotranscobalamin.

disease. However, it only focused on changes in holoTC levels before and after treatment with nutritional supplements containing vitamin B12; there was no comparison with healthy controls, the study sample was relatively small (45 patients), and the disease severity or functional outcomes were not assessed [6]. They concluded that the holoTC level was a promising early diagnostic marker of vitamin B12 deficiency, although there was no statistically significant difference in holoTC values reported at the baseline and after vitamin B12 supplementation. The present study showed that serum holoTC levels, holoTC/total B12 ratios, and homocysteine levels differed significantly between ischemic stroke patients and healthy controls. Moreover, the multivariable logistic regression analysis revealed that holoTC/total B12 ratios and homocysteine levels may be used as predictive factors for the development of ischemic stroke. Taken together, our findings implied that holoTC and total B12 levels could be valuable biomarkers for predicting ischemic stroke risk, along with homocysteine and total B12 levels.

Despite ongoing controversy, several findings in the current study may support the use of holoTC over total B12 measurements in patients with first-ever ischemic stroke. First, holoTC levels were lower in patients than in healthy controls, in both male and female subgroups, and homocysteine levels were also significantly higher in stroke patients of both sexes. However, when total B12 levels were considered, these differences were only apparent in men. Second, in stroke patients, homocysteine levels were inversely associated with holoTC levels but not with total B12 levels based on both of Spearman's rank test and multiple linear regression analysis. Finally, among patients with hyperhomocysteinemia, the holoTC level was decreased in around $21.6 \%$ of patients, while the total B12 level was decreased in only $10.8 \%$ of patients. Taken together, holoTC measurements are likely to better reflect homocysteine levels than total B12 measurements. As high levels of homocysteine in ischemic stroke patients can lead to recurrent ischemic stroke events [24], holoTC measurements in ischemic stroke patients may be useful for identifying patients who require vitamin B12 supplementation.

The holoTC/total B12 ratio was an independent predictive factor for ischemic stroke, but holoTC and total B12 levels alone were not. Garrod et al. reported that the holoTC/total B12 ratio was associated with cognitive function in old patients with depressive symptoms, and they concluded that the holoTC/total B12 ratio may better reflect the adequacy of B12 for nervous system function than holoTC or total B12 level alone $[25,26]$. However, ischemic stroke is a vascular event, and we did not find any evidence about the relationship between holoTC/total B12 ratios and 
homocysteine levels. It was plausible that the holoTC/total $\mathrm{B} 12$ ratio reflected $\mathrm{B} 12$ deficiency in the endovascular system more accurately than the total B12 or holoTC level alone, but the exact mechanisms are unclear. The holoTC/total B12 ratio was also inversely correlated to disease severity in the multiple linear regression analyses. Because serum folate and vitamin B6 levels could also affect homocysteine levels [27] and vitamin B12 deficiency has the potency to reduce taurine levels as described above $[9,19,20]$, further investigation with these biomarkers needs to be performed to explore the utility of holoTC/total B12 ratio measurement and its related mechanisms in stroke patients.

Both holoTC (in multiple linear regression analysis and Spearman's rank test) and total B12 (in Spearman's rank test only) levels, but not serum homocysteine levels, showed significant correlations with the NIHSS score. Additionally, quantitative comparisons between subgroups stratified by the NIHSS score showed that the subgroups with NIHSS scores of 21-42 had significantly lower holoTC and total B12 levels than other subgroups, whereas no differences were found with respect to homocysteine levels. Although results of several previous studies are concordant with this result $[28,29]$, most studies concluded that there was a significant correlation between disease severity and homocysteine levels $[2,30,31]$. The reasons for differences between our own findings and those of previous studies and for the correlation of holoTC and vitamin B12 levels, but not homocysteine levels, with disease severity are unclear. One possible cause for these discrepancies is the relatively small number of patients with NIHSS scores $\geq 16$ in this study. Almost all patients $(92 \%)$ had NIHSS scores $\leq 15$, which could have affected the disease severity results. Vitamin B12 levels may have direct effects on the peripheral or central nervous systems, including cellular energetic functions, antioxidative and neuroprotective actions, and both myelin and neurotransmitter synthesis [32]. These could have affected disease severity irrespective of homocysteine levels, even if the effects were small. The dynamics of homocysteine levels during the disease course could be another cause. Homocysteine levels can fluctuate or increase 24 hours after stroke onset [33]. Because homocysteine is an acute phase reactant, the possibility of the acute phase response being responsible for the elevation in serum homocysteine levels in acute stroke patients cannot be ruled out. Thus, it is very important to be careful when interpreting results related to homocysteine levels.

Similar to the NIHSS score, the mRS score at the time of discharge was correlated with holoTC and total B12 levels, but not with homocysteine levels. These findings were concordant with results of most previous studies [2, 34, 35]. Markisic et al. revealed that worse functional outcomes, assessed according to the mRS after a 6-month follow-up, only correlated with total B12 measurements, and hyperhomocysteinemia did not correlate with mRS scores at any follow-up time point [2]. Mizrahi et al. also reported no correlations between homocysteine levels and functional outcomes when the functional independence measure tool was used [35]. Contrary to most studies, one study reported that the combination of homocysteine and high-sensitivity C- reactive protein levels was associated with short-term outcomes of ischemic stroke [36].

This study had several limitations. First, because of the lack of related medical records, we did not assess physical activity, alcohol overuse, unhealthy diet, psychosocial stress, or depression, which are factors known to contribute to ischemic stroke risk [2]. Second, serum levels of vitamin B9 (folic acid) and vitamin B6, which can also affect homocysteine levels, were not measured. In addition, serum levels of cytokines such as interleukins and tumor necrosis factors, which can be confounding factors of homocysteine levels [37], were not measured. They were also known to be related to disease severity [38]. We also did not assess the levels of methylmalonic acid, which is another biomarker affected by vitamin B12 deficiency. Third, we did not investigate point mutations in the coding region of the MTHFR gene, which is associated with mild and moderate hyperhomocysteinemia [39]. Fourth, the number of patients with severe ischemic stroke was relatively small. Finally, the onset time of stroke symptoms mainly depended on patients' or their families' recall; thus, it could not be validated.

\section{Conclusion}

Despite the limitations, our findings suggested that measurements of serum holoTC along with total B12 and homocysteine may provide valuable information for predicting ischemic stroke onset and its severity and short-term outcomes of ischemic stroke patients. Further research is needed for improved validation.

\section{Data Availability}

The data used to support the findings of this study are available from the corresponding author upon request.

\section{Disclosure}

The funder had no role in the writing of the manuscript or the decision to submit it for publication and was not involved in data collection, analysis, or interpretation; trial design; patient recruitment; or any aspect pertaining to the study.

\section{Conflicts of Interest}

The authors have declared that no competing interest exists.

\section{Authors' Contributions}

Research conception and design were done by Kim HR. Data acquisition was done by Lim YK and Kweon OJ. Data analysis and interpretation were done by Kweon OJ and Lim YK. Statistical analysis was done by Lim YK and Kweon OJ. Drafting of the manuscript was done by Kweon OJ. Critical revision of the manuscript was done by Kim $\mathrm{HR}$ and Lee MK. Kim HR was responsible for receiving grant. Approval of the final manuscript was done by all the authors. All authors declare that they take responsibility for the entire content of the manuscript. 


\section{Acknowledgments}

This work was supported by the Basic Science Research Program through the National Research Foundation of Korea (NRF) funded by the Ministry of Education (NRF2017R1A2B4011631 and NRF-2021R1A2C2013359).

\section{References}

[1] G. J. Hankey, “Stroke,” Lancet, vol. 389, no. 10069, pp. 641654, 2017.

[2] M. Markisic, A. M. Pavlovic, and D. M. Pavlovic, "The impact of homocysteine, vitamin B12, and vitamin D levels on functional outcome after first-ever ischaemic stroke," BioMed research international, vol. 2017, Article ID 5489057, 6 pages, 2017.

[3] M. J. O'Donnell, D. Xavier, L. Liu et al., "Risk factors for ischaemic and intracerebral haemorrhagic stroke in 22 countries (the INTERSTROKE study): a case-control study," Lancet, vol. 376, no. 9735, pp. 112-123, 2010.

[4] K. S. Woo, K. E. Kim, J. S. Park, J. I. Park, and J. Y. Han, "Relationship between the levels of holotranscobalamin and vitamin B12," The Korean journal of laboratory medicine, vol. 30, no. 2, pp. 185-189, 2010.

[5] G. J. Hankey, "B vitamins for stroke prevention," Stroke and vascular neurology, vol. 3, no. 2, pp. 51-58, 2018.

[6] S. Serefhanoglu, I. Aydogdu, E. Kekilli, A. Ilhan, and I. Kuku, "Measuring holotranscobalamin II, an early indicator of negative vitamin B12 balance, by radioimmunoassay in patients with ischemic cerebrovascular disease," Annals of hematology, vol. 87, no. 5, pp. 391-395, 2008.

[7] W. Herrmann and R. Obeid, "Cobalamin deficiency," Sub-cellular biochemistry, vol. 56, pp. 301-322, 2012.

[8] G. Saposnik, "The role of vitamin B in stroke prevention: a journey from observational studies to clinical trials and critique of the VITAmins TO Prevent Stroke (VITATOPS)," Stroke, vol. 42, no. 3, pp. 838-842, 2011, Epub 2011/01/29.

[9] J. D. Spence, "Metabolic vitamin B12 deficiency: a missed opportunity to prevent dementia and stroke," Nutrition research, vol. 36, no. 2, pp. 109-116, 2016, Epub 2015/11/26.

[10] E. Nexo and E. Hoffmann-Lucke, "Holotranscobalamin, a marker of vitamin B-12 status: analytical aspects and clinical utility," The American journal of clinical nutrition, vol. 94, no. 1, pp. 359s-365s, 2011.

[11] P. H. Golding, "Holotranscobalamin (HoloTC, active-B12) and Herbert's model for the development of vitamin B12 deficiency: a review and alternative hypothesis," Springerplus, vol. 5, no. 1, p. 668, 2016.

[12] V. Devalia, M. S. Hamilton, and A. M. Molloy, "Guidelines for the diagnosis and treatment of cobalamin and folate disorders," British journal of haematology., vol. 166, no. 4, pp. 496-513, 2014.

[13] R. L. Sacco, S. E. Kasner, J. P. Broderick et al., "An updated definition of stroke for the 21st century: a statement for healthcare professionals from the American Heart Association/American Stroke Association," Stroke, vol. 44, no. 7, pp. 2064-2089, 2013.

[14] P. Ganguly and S. F. Alam, "Role of homocysteine in the development of cardiovascular disease," Nutrition journal, vol. 14, no. 1, p. $6,2015$.
[15] S. Zhang, Y. Y. Bai, L. M. Luo, W. K. Xiao, H. M. Wu, and P. Ye, "Association between serum homocysteine and arterial stiffness in elderly: a community-based study," Journal of geriatric cardiology, vol. 11, no. 1, pp. 32-38, 2014.

[16] A. Baszczuk and Z. Kopczynski, "[Hyperhomocysteinemia in patients with cardiovascular disease]. Postepy higieny i medycyny doswiadczalnej (Online)," Hiperhomocysteinemia $u$ chorych na schorzenia ukladu krazenia, vol. 68, pp. 579589, 2014.

[17] D. S. Wald, M. Law, and J. K. Morris, "Homocysteine and cardiovascular disease: evidence on causality from a meta-analysis," BMJ, vol. 325, no. 7374, pp. 1202-1206, 2002.

[18] Homocysteine Studies Collaboration and Homocysteine Studies Collaboration, "Homocysteine and risk of ischemic heart disease and Stroke," Jama, vol. 288, no. 16, pp. 2015-2022, 2002.

[19] S. Murakami, "Taurine and atherosclerosis," Amino Acids, vol. 46, no. 1, pp. 73-80, 2014.

[20] P. Roman-Garcia, I. Quiros-Gonzalez, L. Mottram et al., "Vitamin B12-dependent taurine synthesis regulates growth and bone mass," The Journal of clinical investigation, vol. 124, no. 7, pp. 2988-3002, 2014.

[21] M. Vogeser and S. Lorenzl, "Comparison of automated assays for the determination of vitamin B12 in serum," Clinical Biochemistry, vol. 40, no. 16-17, pp. 1342-1345, 2007.

[22] V. Devalia, "Diagnosing vitamin B-12 deficiency on the basis of serum B-12 assay," BMJ, vol. 333, no. 7564, pp. 385-386, 2006.

[23] M. Ulleland, I. Eilertsen, E. V. Quadros et al., "Direct assay for cobalamin bound to transcobalamin (holo-transcobalamin) in serum," Clinical chemistry, vol. 48, no. 3, pp. 526-532, 2002.

[24] M. Zhao, X. Wang, M. He et al., "Homocysteine and stroke risk," Stroke, vol. 48, no. 5, pp. 1183-1190, 2017.

[25] M. G. Garrod, R. Green, L. H. Allen et al., "Fraction of total plasma vitamin B12 bound to transcobalamin correlates with cognitive function in elderly Latinos with depressive symptoms," Clinical chemistry, vol. 54, no. 7, pp. 1210-1217, 2008.

[26] M. G. Garrod, R. Green, L. H. Allen et al., "The ratio of holotranscobalamin to total $\mathrm{B} 12$ is associated with cognitive impairment in elderly Latinos with elevated depression scores," The FASEB Journal, vol. 21, no. 5, 2007.

[27] E. Lonn, S. Yusuf, M. J. Arnold et al., "Homocysteine lowering with folic acid and B vitamins in vascular disease," The New England journal of medicine, vol. 354, no. 15, pp. 1567-1577, 2006.

[28] F. Perini, E. Galloni, I. Bolgan et al., "Elevated plasma homocysteine in acute stroke was not associated with severity and outcome: stronger association with small artery disease," Neurological Sciences., vol. 26, no. 5, pp. 310-318, 2005.

[29] H. Suleiman, I. Aliyu, S. Abubakar et al., "Assessment of homocysteine, vitamin $\mathrm{B}_{12}$, and zinc levels among patients with acute ischemic stroke in Northwestern Nigeria," Nigerian Journal of Basic and Clinical Sciences, vol. 14, no. 2, pp. 105108, 2017.

[30] R. Bonthapally, R. Jacob, K. Baba, R. Kandadai, and I. M. N. Mohan, "Association between homocysteine and stroke severity," IOSR Journal of Dental and Medical Sciences, vol. 16, pp. 13-16, 2017.

[31] S. Harris, A. Rasyid, M. Kurniawan, T. Mesiano, and R. Hidayat, "Association of high blood homocysteine and risk 
of increased severity of ischemic stroke events," The International journal of angiology, vol. 28, no. 1, pp. 34-38, 2019.

[32] C. A. Calderón-Ospina and M. O. Nava-Mesa, "B vitamins in the nervous system: current knowledge of the biochemical modes of action and synergies of thiamine, pyridoxine, and cobalamin," CNS Neuroscience \& Therapeutics, vol. 26, no. 1, pp. 5-13, 2020.

[33] V. J. Howard, E. G. Sides, G. C. Newman et al., "Changes in plasma homocyst(e)ine in the acute phase after stroke," Stroke, vol. 33, no. 2, pp. 473-478, 2002.

[34] W. Wang, C. Gao, C. Yu et al., "No association between elevated total homocysteine levels and functional outcome in elderly patients with acute cerebral infarction," Frontiers in aging neuroscience, vol. 9, 2017.

[35] E. H. Mizrahi, Y. Fleissig, M. Arad, and A. Adunsky, "Plasma homocysteine level and functional outcome of patients with ischemic stroke," Archives of physical medicine and rehabilitation, vol. 86, no. 1, pp. 60-63, 2005.

[36] W. J. Tu, S. J. Zhao, T. G. Liu, D. G. Yang, and H. Chen, "Combination of high-sensitivity C-reactive protein and homocysteine predicts the short-term outcomes of Chinese patients with acute ischemic stroke," Neurological Research, vol. 35, no. 9, pp. 912-921, 2013.

[37] A. M. Gori, A. M. Corsi, S. Fedi et al., "A proinflammatory state is associated with hyperhomocysteinemia in the elderly," The American journal of clinical nutrition., vol. 82, no. 2, pp. 335-341, 2005.

[38] O. A. Sobowale, A. R. Parry-Jones, C. J. Smith, P. J. Tyrrell, N. J. Rothwell, and S. M. Allan, "Interleukin-1 in stroke: from bench to bedside," Stroke, vol. 47, no. 8, pp. 2160-2167, 2016.

[39] G. J. Hankey and J. W. Eikelboom, "Homocysteine and vascular disease,” Lancet, vol. 354, no. 9176, pp. 407-413, 1999. 